\title{
Konstitutionelle Gemeinwohlorientierung im Völkerrecht
}

Tobias Stoll*

Rezension zu: Martin Scheyli, Konstitutionelle Gemeinwohlorientierung im Völkerrecht. Grundlagen völkerrechtlicher Konstitutionalisierung am Beispiel des Schutzes der globalen Umwelt, Berlin (Duncker \& Humblot ff Schriften zum Völkerrecht, Bd. 185) 2008, 572 S., $78 €$, ISBN 978-3-428-12826-6

In seiner großen Monographie behandelt der Schweizer Völkerrechtler Martin Sche$y l i$, eine aktuelle Kernfrage der Völkerrechtswissenschaft, nämlich die der Konstitutionalisierung. Inwieweit das Völkerrecht mit seinen Entwicklungen sich als Verfassungsordnung - vielleicht auch nur als entstehende Verfassungsordnung - beschreiben lässt, wird seit einiger Zeit breit diskutiert. Seitdem die bipolare Ordnung der Welt durch zwei Supermächte mit dem Fall der Berliner Mauer zu Ende gegangen ist, stellt sich deutlich die Frage nach der Ordnungsleistung des Völkerrechts und nach dem Charakter dieser Rechtsordnung. Ob und inwieweit man jenseits nationalstaatlicher Ordnungen von einer Verfassung sprechen kann, ist die Kernfrage, der Scheyli in seiner umfangreichen Untersuchung nachgeht. Die Besonderheit der Arbeit liegt darin, dass sie den Begriff der Konstitutionalisierung auf das Gemeinwohl orientiert und für ihre Erkenntniszwecke als Beispiel den internationalen Umweltschutz herausgreift. Im Ergebnis hält Scheyli es für möglich und wohl auch sinnvoll, im Völkerrecht von einer Konstitutionalisierung zu sprechen, sofern es „ein Minimum konstitutioneller Realität“ (S. 198 f.) gibt, das sich durch die Bestimmung eines allgemeinen Wohls, die entsprechende Herausprägung normativer Grundentscheidungen und „konstitutioneller Normen“ beschreiben ließe, wobei weiterhin zu fordern sei, dass diese konstitutionellen Normen durchgesetzt bzw. beachtet würden. Aufgrund dieses Maßstabes gelangt er zu einer differenzierten Betrachtung des konstitutionellen Gehalts des internationalen Umweltrechts.

Scheyli beginnt seine Untersuchung mit einem schönen Überblick über die Entwicklung des Völkerrechts hin zu einer universellen Rechtsordnung (Kapitel 1). Mit sicherem Zugriff wird die Entwicklung des universellen Völkerrechts aus dem europäischen System mit ihrer Deutung durch die großen klassischen Theoretiker des Völkerrechts beschrieben. Kurz und prägnant wird auf wesentliche Determinanten dieser Völkerrechtsordnung in Form der Einschränkung der vormals absoluten Souveränität und des Übergangs von der Koordination zur Kooperation hingewiesen.

In einem zweiten Kapitel entfaltet Scheyli dann den Begriff der Konstitutionalisierung und die damit verbundenen Erkenntnis- und Deutungsinteressen in der Völkerrechtslehre. Er weist darauf hin, dass die Globalisierung und die damit verbundenen

* Prof. Dr. Tobias Stoll lehrt Öffentliches Recht, insbesondere Völkerrecht und Internationales Wirtschafts- und Umweltrecht an der Georg-August-Universität Göttingen. 
Herausforderungen der Ausgangspunkt dieser Diskussion waren und differenziert dann verschiedene Sichtweisen und Ansätze einer solchen Konstitutionalisierung im Völkerrecht. Dabei wird unter zutreffender Betonung der Unterschiedlichkeiten auf die europäische Verfassungsfrage eingegangen (S. 126 ff.). In einer vergleichenden Betrachtung schildert Scheyli dann, unter welchen Umständen man auf nationaler, supernationaler und internationaler Ebene von Verfassung sprechen kann. Scheyli räumt dabei ein, dass der Verfassungsbegriff, wenn er auf die internationale Ebene bezogen sein soll, deutlich reduziert werden muss (S. $191 \mathrm{ff}$.) und dass er ein „konzeptionelles“ Begriffsverständnis zugrunde legt. Als These wird insofern formuliert: "Was auf der völkerrechtlichen Ordnungsebene als (reduziertes) Kriterium der Verfassungsfähigkeit der sich konstituierenden Gemeinschaft in Frage kommt, ist die Fähigkeit, das Gemeinwohl zu definieren, entsprechende normative Grundentscheidungen zu treffen, aus diesem konstitutionelle Normen zu entwickeln und schließlich für deren Durchsetzung besorgt zu sein." (S. 199)

In einem dritten großen theoretischen Kapitel (S. $203 \mathrm{ff}$.) wird dann der in dieser Grundthese zentrale Begriff des Gemeinwohls entfaltet. Scheyli setzt sich dabei zunächst mit den Grundlagen einer solchen Gemeinwohlorientierung auseinander und verweist dabei insbesondere auf gemeinsame Interessen und soziale Grundlagen des Völkerrechts (S. 204 ff.). Anschließend wird die Bestimmung dieses Gemeinwohls mit den damit zusammenhängenden Legitimationsfragen diskutiert (S.231 ff.). Schließlich wird in inhaltlicher Hinsicht das Gemeinwohl von einer „internationalen Solidarität“ (S. 258 ff.) abgegrenzt. Es schließt sich eine Betrachtung der verschiedenen Normkategorien des Völkerrechts an, in der Scheyli auf die besondere Bedeutung des Völkergewohnheitsrechts einerseits und von Prinzipienbildungen im Völkerrecht hinweist. Er versteht dabei solche Prinzipien als Normen und lässt erkennen, dass er solchen Prinzipien insbesondere die Qualität von Verfassungsnormen zuschreibt.

Es schließt sich im vierten Kapitel eine „Probe auf Exempel“ an: Scheyli bezieht jetzt den zuvor entwickelten Ansatz auf das Umweltvölkerrecht. Dabei konzentriert er sich zunächst in einer allgemeinen Betrachtung auf die im Umweltvölkerrecht spätestens seit der Rio-Konferenz von 1992 intensiv betriebenen Prinzipienbildung, die seinem Verständnis von Konstitutionalisierung stark entgegen kommt. Anschließend wird aus dieser Perspektive das internationale Klimaschutzrecht untersucht. Das Ergebnis ist ernüchternd. Scheyli kommt zu dem Ergebnis, dass die geringen Fortschritte bei der rechtlich verbindlichen Regelung bei der internationalen Zusammenarbeit im Hinblick auf den Schutz vor dem Klimawandel den Anforderungen der zuvor entwickelten Prinzipien nicht gerecht werden. Dies gilt insbesondere für das Vorsorgeprinzip (S. 444).

Dieses Ergebnis wird in einem fünften Kapitel ausführlich gewürdigt, das mit den Worten „Hindernisse und Entwicklungsperspektiven völkerrechtlicher Konstitutio- 
nalisierung “ (S. 448 ff.) überschrieben ist. Hier weist Scheyli zunächst darauf hin, dass sich in den internationalen Beziehungen zum Teil eine Tendenz zu unilateralem Handeln abzeichnet, andererseits aber große Herausforderungen für ein gemeinsames Handeln - gerade im Bereich des Klimaschutzes - stellen. Im Anschluss daran umreißt Scheyli kurz die Bedingungen, die für die Ausprägung einer zumindest teilweisen, auf den Umweltschutz bezogenen Verfassungsordnung auf internationaler Ebene erforderlich sei. Dazu gehört für ihn eine Weiterentwicklung völkerrechtlicher Normen im Hinblick auf ihren zwingenden Charakter bzw. die Bindung von Staaten, die diesen Normen nicht zugestimmt haben. Schließlich gehört dazu nach Scheyli die Bereitschaft der Staaten, zur Verwirklichung von Gemeinwohlzielen die Reziprozitätserwartungen in ihrem außenpolitischen Handeln zurückzustellen und ein entsprechend eingeschränktes neues Souveränitätsverständnis.

Gerade mit ihren einführenden Teilen macht die Untersuchung deutlich, in welcher großen Traditionslinie die aktuelle Diskussion in der Völkerrechtslehre über die „Konstitutionalisierung “ steht. Die ganze Entwicklung des Völkerrechts ist von Vorstellungen begleitet worden, dass hinter der Regelung der Verhältnisse zwischen souveränen Staaten durch Gewohnheit oder Vertrag ein größerer ordnender Zusammenhang steht, der sich auf ein Element der Gemeinschaftlichkeit und eines allgemeinen Wohls stützt und normative Bedeutung hat. Den vielen eindrucksvollen Betrachtungen zu diesem Thema ist gemein, dass nie ganz deutlich wird, ob dieser ordnende Zusammenhang ein Desiderat darstellt oder zumindest in Teilen schon verwirklicht ist.

Auch die Untersuchung von Scheyli ist mit ihren Ergebnissen in dieser Zwischenwelt zwischen Wunsch und Wirklichkeit anzusiedeln. Sie immer neu zu vermessen, ist ein legitimes und wichtiges Anliegen der Völkerrechtslehre, die nicht nur nach dem Bestehenden, sondern auch nach der Zukunft fragt. Scheyli ist darin zuzustimmen, dass man dies auch mit dem Begriff der Verfassung tun kann. Das ist den wenigen aber deutlichen deutschen Stimmen entgegen zu halten, die jenseits des nationalstaatlichen Bereichs jede Verwendung des Begriffs der Verfassung am liebsten verbieten würden.

Die Perspektive der Verfassung wird in der Völkerrechtslehre - wie Scheyli sorgfältig und zu Recht ausführt - „konzeptionell“, d. h. analytisch, verwendet. So gesehen ist die Verwendung des Begriffs danach zu beurteilen, inwieweit damit eine sinnvolle analytische Kategorie definiert werden kann. Das hängt vor allem davon ab, welcher Erkenntnisertrag mit einer solchen Bezeichnung verbunden ist. Ein solcher Ertrag mag in dem Vergleich nationaler Verfassungsordnungen mit ihren vielfältigen Elementen und Leistungen einerseits und der Grundstrukturen der Völkerrechtsordnung andererseits liegen. Darauf geht Scheyli in seiner Untersuchung zu Recht ein. Sein Interesse an der Kategorie der Verfassung liegt aber - wie oben schon angedeutet worden ist - darin, im Völkerrecht auf einer übergeordneten Ebene einen Ordnungs- 
zusammenhang mit normativem Gehalt zu identifizieren. Die Untersuchung von Scheyli zeigt die Mühe und Schwierigkeiten, eine solche Struktur mit besonderer Dignität zu beschreiben, die die Verwendung des Begriffs der Verfassung rechtfertigen könnte. Mit dem Hinweis auf das „reduzierte“ Verständnis von Verfassung ist dies ansatzweise umschrieben.

Die oben erwähnte "Grundthese“ von Scheyli beschreibt letztlich eine relativ anspruchslose „Verfassungsqualität“. Ihre Anwendung in dem Bereich des Umweltvölkerrechts, das im Hinblick auf die Anerkennung eines Gemeinwohls, die Herausprägung von Prinzipien und die Kooperationsdimension als besonders fortschrittlicher Teil des Völkerrechts angesehen werden kann, ist ernüchternd und auf der anderen Seite möglicherweise irreführend: Auch Nationalstaaten, an deren Verfasstheit wir keinen Zweifel haben, lassen sich trotz zum Teil bestehender verfassungsrechtlicher Gebote mitunter viel Zeit, bevor sie die Herausforderungen des Umweltschutzes wahrnehmen und dementsprechend handeln. Darüber hinaus ist problematisch, dass Scheyli hier einen Verfassungsgehalt deswegen als naheliegend erachtet, weil er den völkerrechtlichen Umweltprinzipien eine sehr starke normative Stellung einräumt. Ob solche Prinzipienbildungen notwendigerweise zum Inbegriff von Verfassung - auf staatlicher wie auf internationaler Ebene - gehört, ist zu bezweifeln.

Auf sehr viel grundsätzlicherer Ebene sind auch Zweifel im Hinblick auf das Vorhaben angebracht, über eine konstitutionelle Substanz mit Blick allein auf das Umweltrecht zu sprechen. Wenn Scheyli eine „Verfassungssubstanz“ allein des Umweltvölkerrechts für möglich hält und daneben an anderer Stelle in seiner Untersuchung von der konstitutionellen Qualität des Welthandelsrechts und des europäischen Menschenrechtsschutzes spricht, so liegt darin die Vorstellung, dass wir über Teilsysteme von Rechtsordnungen sinnvoll in der Kategorie von Verfassung sprechen könnten. Das wird allerdings in der Untersuchung nicht mit der gebotenen Deutlichkeit diskutiert. Es liegt nämlich das Argument auf der Hand, dass wir möglicherweise mit dem Begriff der Verfassung gerade diesen umfassenden, sachübergreifenden Geltungsanspruch einer normativen Herrschaftsordnung verbinden müssen. Diese Anmerkungen mindern aber die Bedeutung und den Gewinn der Abhandlung nicht. Sie ist in Anbetracht der Herausforderungen, die mit der Fragestellung verbunden sind, als ein wichtiger Beitrag zu verstehen. 